\title{
Enhanced NIF Neutron Activation Diagnostics
}

C. B. Yeamans, D. L. Bleuel, L. A. Bernstein

May 9, 2012

High Temeperature Plasma Diagnostics Monterey, CA, United States

May 7, 2012 through May 10, 2012 
This document was prepared as an account of work sponsored by an agency of the United States government. Neither the United States government nor Lawrence Livermore National Security, LLC, nor any of their employees makes any warranty, expressed or implied, or assumes any legal liability or responsibility for the accuracy, completeness, or usefulness of any information, apparatus, product, or process disclosed, or represents that its use would not infringe privately owned rights. Reference herein to any specific commercial product, process, or service by trade name, trademark, manufacturer, or otherwise does not necessarily constitute or imply its endorsement, recommendation, or favoring by the United States government or Lawrence Livermore National Security, LLC. The views and opinions of authors expressed herein do not necessarily state or reflect those of the United States government or Lawrence Livermore National Security, LLC, and shall not be used for advertising or product endorsement purposes. 


\section{NIF}

June 29, 2012

Charles Yeamans

PO Box 808, L-550

Livermore, CA 94550

Subject: Response Letter, RSI-HTPD MS\# C121076-P.5.10R

Dear Dr. Young:

Comments provided by Reviewer \#1 were largely incorporated as suggested into the submission, with the only omissions being made for space. Sections were re-ordered for clarity and continuity. Specific reviewer comments were addressed in the following major changes:

1) Section III.B Potassium Zirconate Composite Detector was expanded to include discussion of competing and interfering reactions as suggested. The section now also justified and makes explicit the selection of lanthanum bromide scintillators, as the reviewer commented had been indeterminate in the original text.

2) Section VI. Engineering Considerations was expanded to include more discussion of detector physical geometry design.

Minor revisions to illustrations were:

1) Labeling of axes in Figure 1

2) Modifying legend aspect ratio and adding gridlines to Figure 2.

3) Reordering of Figures 3 and 4.

Overall, the reviewers comments were extremely helpful in revising the submission.

Sincerely,

Charles Yeamans

postdoc

National Ignition Facility Programs Directorate 


\title{
Enhanced NIF Neutron Activation Diagnostics ${ }^{\text {a) }}$
}

\author{
C. B. Yeamans, ${ }^{1, b}$ D. L. Bleuel, ${ }^{1}$ and L. A. Bernstein ${ }^{1}$ \\ ${ }^{\prime}$ Lawrence Livermore National Laboratory, Livermore, CA, 94550 \\ (Presented XXXXX; received XXXXX; accepted XXXXX; published online XXXXX)
}

The NIF neutron activation diagnostic suite relies on removable activation samples, leading to operational inefficiencies and a fundamental lower limit on the half-life of the activated product that can be observed. A neutron diagnostic system measuring activation of permanently-installed samples could remove these limitations and significantly enhance overall neutron diagnostic capabilities. The physics and engineering aspects of two proposed systems are considered: one measuring the ${ }^{89} \mathrm{Zr}{ }^{89 \mathrm{~m}} \mathrm{Zr}$ isomer ratio in the existing $\mathrm{Zr}$ activation medium and the other using potassium zirconate as the activation medium. Both proposed systems could improve the signal-to-noise ratio of the current system by at least a factor of 5 and would allow independent measurement of fusion core velocity and fuel areal density.

\section{INTRODUCTION}

NIF neutron yields are measured by a suite of neutron activation diagnostics (NAD) utilizing the activation and subsequent counting of target material placed in and/or around the NIF target chamber ${ }^{1}$. The ${ }^{115} \operatorname{In}\left(n, n^{\prime}\right){ }^{115-m}$ In reaction measures $\mathrm{D}(\mathrm{D}, \mathrm{n})$ neutron yield and both ${ }^{90} \mathrm{Zr}(\mathrm{n}, 2 \mathrm{n}){ }^{89} \mathrm{Zr}$ and ${ }^{63} \mathrm{Cu}(\mathrm{n}, 2 \mathrm{n}){ }^{62} \mathrm{Cu}$ are used for $D(T, n)$ yield measurements ${ }^{2,3}$. Activation diagnostics are considered one of the primary neutron yield measurements because they produce results independent of any other diagnostic system. Measurements from other diagnostics are calibrated to activation results.

NIF neutron yields are calculated from the measured activation sample activity by:

$$
Y_{D T}=\frac{A_{0}}{p_{r x} \cdot \varepsilon_{i r r}}
$$

where

$Y_{D T}$ : primary DT neutron yield

$A_{0}$ : initial activity product nuclei formed at $\mathrm{t}=0$

$p_{r x}$ : reaction probability with activation foil per neutron born at target chamber center (TCC)

$\varepsilon_{i r r}$ irradiation efficiency, fraction of neutrons born at TCC within subtended solid angle reaching location of activation foil

The reaction probability per primary neutron is calculated as:

$$
p_{r x}=\frac{N_{\text {target }}}{4 \pi R^{2}} \int_{E} \frac{d n}{d E} \sigma(E) d E
$$

where

$N_{\text {target }}:$ number of target nuclei in activation foil

$R$ : radius of foil from TCC

$n$ : neutrons emitted at TCC
A modeled Gaussian neutron spectrum $(\mu=14.07 \mathrm{MeV}$, $\sigma=0.150 \mathrm{MeV}$ ) is used for $d n / d E$ in both the NIF Flange-NAD analysis and in this paper to evaluate potential design improvements. Though this represents a simplification from the measured neutron spectrum, because of the relatively high thresholds for useful activation reactions, the effect on activation measurements is small in magnitude and consistent over all shots.

In addition to measuring absolute yield, the Flange-NAD system measures relative spatial distribution of unscattered primary neutrons reaching the target chamber wall through the ${ }^{90} \mathrm{Zr}(\mathrm{n}, 2 \mathrm{n}){ }^{89} \mathrm{Zr}$ reaction at 17 locations (FIG. 1). Relative differences in activation are interpreted, per Equation $l$ and Equation 2, as the result of either:

1) A bulk center-of-mass velocity of the burning core Doppler shifting the neutron spectrum, thus changing the spectrum-averaged activation cross section as the velocity vector projection changes with observation position

2) Differences in interstitial scattering mass, such as capsule areal fuel density, creating a spatial variation in the number of neutrons impinging on activation foils around the chamber

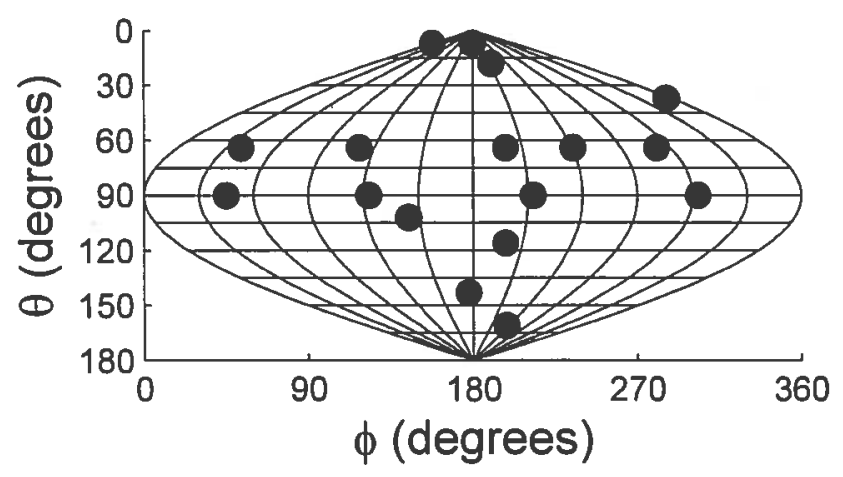

FIG.I. NIF Flange-NAD deployment. Zirconium activation samples are placed on exterior blind flanges around the NIF target chamber, removed post-shot, and analyzed for ${ }^{89} \mathrm{Zr}$ induced activity.

a) Contributed paper published as part of the Proceedings of the 19th Topical Conference on High-Temperature Plasma Diagnostics, Monterey, California, May, 2012.

b)Author to whom correspondence should be addressed: yeamans! @IInl.gov. 
The signal from a single NAD position can be characterized by the difference in activation between a measurement and a reference. In the case of a core velocity measurement, two locations opposed $180^{\circ}$ through TCC see the same velocity magnitude with opposite direction (Equation 3). The magnitude of $\Delta A_{0}$ for the ${ }^{90} \mathrm{Zr}(\mathrm{n}, 2 \mathrm{n})^{89} \mathrm{Zr}$ reaction is not likely to exceed $15 \%$ for plausible core velocities.

$$
\Delta A_{0}=\frac{A_{0}(+v)-A_{0}(-v)}{A_{0}(v=0)}
$$

A significant limitation of the current implementation is the relative sensitivity of the ${ }^{90} \mathrm{Zr}(n, 2 n){ }^{89} \mathrm{Zr}$ and ${ }^{63} \mathrm{Cu}(n, 2 n){ }^{62} \mathrm{Cu}$ reactions to both core velocity and differential scattering, leading to difficulty in mapping activation at the target chamber wall to fuel geometry. Additionally, the reliance on removable activation samples leads to a fundamental lower limit on the half-life of the activated product. The NIF neutron diagnostic capability would be greatly improved by developing a NAD system utilizing an activation medium with more advantageous nuclear properties and a detector system integrated with the activation medium and installed on or near the target chamber.

Two potential improvements are considered:

1) Zirconium Isomeric Ratio Detector: Installing detectors at existing zirconium activation locations, measuring the isomeric ratio of ${ }^{89} \mathrm{Zr}{ }^{89 m} \mathrm{Zr}$ through the relative intensities of the $909 \mathrm{keV}$ and $588 \mathrm{keV}$ gamma rays

2) Potassium Zirconate Composite Detector: Using potassium zirconate $^{4} \quad\left(\mathrm{~K}_{2} \mathrm{ZrO}_{3}\right)$ as the activation medium, measuring the induced activity of ${ }^{38} \mathrm{~K}$ through the $2178 \mathrm{keV}$ gamma ray

\section{PHYSICS DESIGN CONSIDERATIONS}

Activation medium selected must have sufficient activation cross section, with a known low uncertainty, to induce measureable activation at current and anticipated NIF yields. For practical nuclear counting, a minimum of $10^{5}$ activations per detector must be produced in the activation volume. Additionally, the energy threshold of the activation reaction must be high enough to discriminate unscattered primary neutrons from lowangle downscattered neutrons. Entry channels, half-life, gamma energy and gamma branching ratios of the activation product must be considered in materials selection and configuration of the counting system.

The greatest advantage of installed detectors is the ability to measure activation products with half-lives too short to be measured practically with physical removal and counting. The current activation reaction includes a channel to ${ }^{89 \mathrm{~m}} \mathrm{Zr}^{5,6}$. In the current implementation, the 4.16 minute half-life is too short to measure, so all information is taken from the decay of the ${ }^{89} \mathrm{Zr}$ ground state. However, the metastable isomer could be measured in conjunction with the ground state in an installed detector configuration. Additionally, the ${ }^{39} \mathrm{~K}(\mathrm{n}, 2 \mathrm{n}){ }^{38} \mathrm{~K}$ reaction ${ }^{7}$ has many of the same attractive properties as the currently-employed ${ }^{90} \mathrm{Zr}(\mathrm{n}, 2 \mathrm{n})^{89} \mathrm{Zr}$ reaction, ${ }^{8}$, with the added benefit of a higher threshold. With a threshold of $13.42 \mathrm{MeV}$ and a half-life of 7.676 minutes, ${ }^{39} \mathrm{~K}(\mathrm{n}, 2 \mathrm{n}){ }^{38} \mathrm{~K}$ allows the highest energy discrimination of primary neutrons from scattered neutrons available in a material meeting the mechanical and chemical design criteria. Figure 2 shows cross sections for activation reactions of interest with a thermal NIF DT spectrum superimposed for reference.

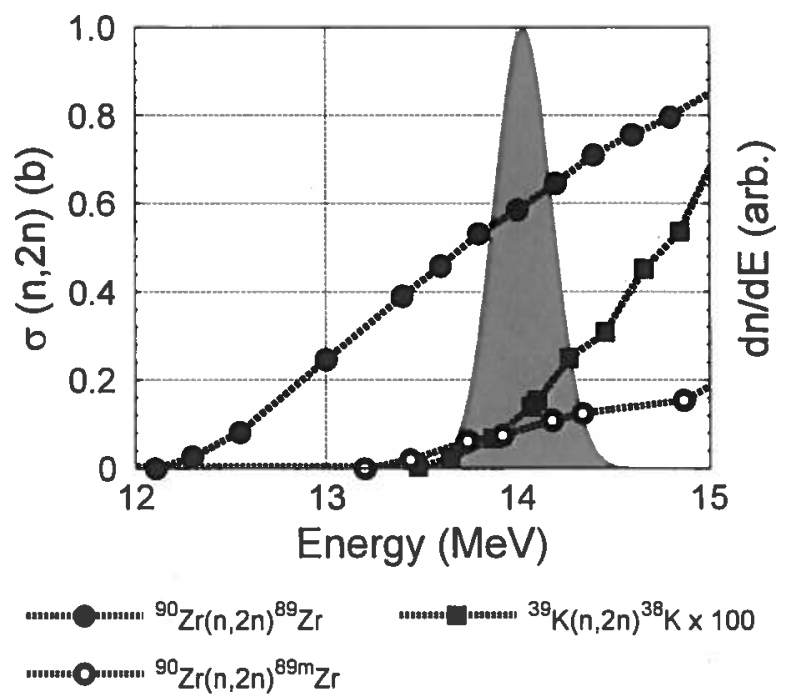

FIG. 2. Cross section data for activation reactions $s^{5,6,7,8}$ in proposed NAD improvements, with the modeled DT spectrum used in the activation calculation superimposed for reference.

\section{CALCULATED DETECTOR RESPONSE}

\section{A. Zirconium Isomeric Ratio Detector}

The ${ }^{90} \mathrm{Zr}(\mathrm{n}, 2 \mathrm{n})$ reaction populates ${ }^{89 \mathrm{~m}} \mathrm{Zr}$ in an isomeric ratio that changes significantly over the $13-15 \mathrm{MeV}$ region of interest for DT fusion neutron diagnostics. By measuring the ${ }^{89} \mathrm{Zr}{ }^{89 \mathrm{~m}} \mathrm{Zr}$ activity ratio as well as the ${ }^{89} \mathrm{Zr}$ activity, the neutron energy can be deconvolved from the number of neutrons. Peak Doppler shifts observed in NIF shots can drive a significant fraction of the primary neutron spectrum below the ${ }^{90} \mathrm{Zr}(n, 2 n)^{89 \mathrm{~m}} \mathrm{Zr}$ threshold.

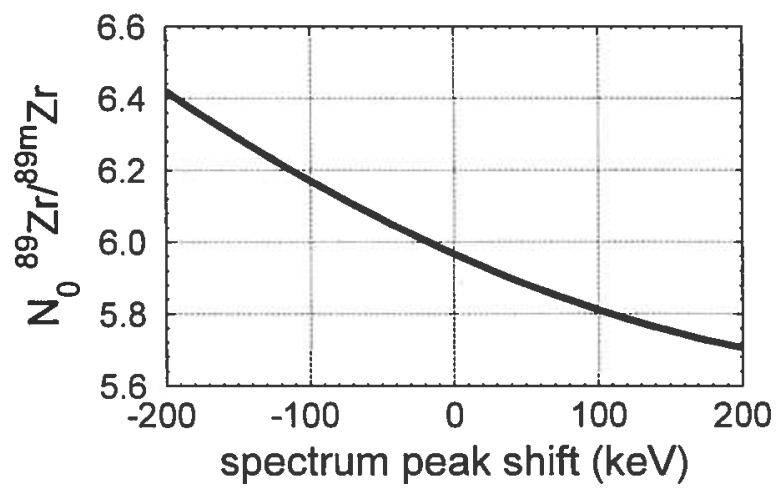

FIG. 3. Zirconium isomer ratio as a function of spectrum peak shift. The isomer ratio changes substantially over the region of interest for DT fusion neutron diagnostics. An idealized Gaussian neutron spectrum $(\mu=14.07 \mathrm{MeV}, \sigma=0.150 \mathrm{MeV})$ is used for activation calculations. 
Figure 3 shows ${ }^{89} \mathrm{Zr}{ }^{89 m} \mathrm{Zr}$ isomeric ratio formed by integrating the respective cross sections across a Doppler-shifted thermal DT fusion spectrum as a function of the energy shift. The activation signal is similar to that obtained in the ${ }^{90} \mathrm{Zr}(n, 2 n)^{89} \mathrm{Zr}$ measurement, but with the advantage of allowing a spectrum with a Doppler-shifted peak energy to be distinguished from an unshifted spectrum with a change in magnitude.

\section{B. Potassium Zirconate Composite Detector}

Calculating difference in activity between opposed locations using Equation 2 and Equation 3 gives $5.5 \%$ signal per $100 \mathrm{~km} / \mathrm{s}$ core velocity for the ${ }^{90} \mathrm{Zr}(\mathrm{n}, 2 \mathrm{n})^{89} \mathrm{Zr}$ reaction and $31.2 \%$ signal per $100 \mathrm{~km} / \mathrm{s}$ core velocity for the ${ }^{39} \mathrm{~K}(\mathrm{n}, 2 \mathrm{n}){ }^{38} \mathrm{~K}$ reaction, a factor 5.7 improvement in signal-to-background ratio. At typical effective noise levels created by counting statistics and peak fitting errors of $2.75 \%$, the effective signal-to-noise ratio for ${ }^{90} \mathrm{Zr}(n, 2 n)^{89} \mathrm{Zr}$ is $2.0 \mathrm{per} 100 \mathrm{~km} / \mathrm{s}$ core velocity, improved to 11.3 by measuring the ${ }^{39} \mathrm{~K}(\mathrm{n}, 2 \mathrm{n})^{38} \mathrm{~K}$ activation reaction product. Figure 4 shows the response of the two activation media as a function of core velocity. Separation of the effect of core velocity from that of differential scattering may be accomplished by first determining core velocity from the relative values of potassium activation and Equation 3, then refining $d n / d E$ when calculating neutron fluence from Equation 2 and the measured zirconium activation. The two parameters of core velocity and spectrum peak height may be varied to obtain an iterative solution for $d n / d E$.

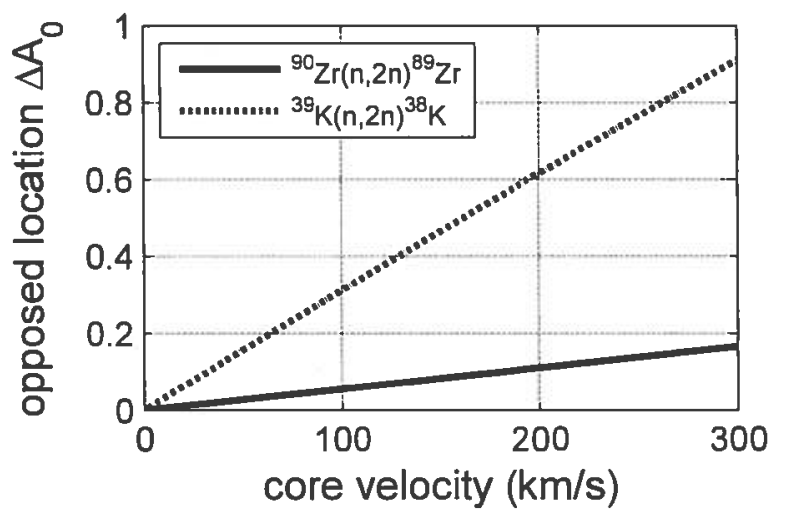

FIG. 4. Activation difference in opposed NAD positions as a function of fusion core bulk velocity. Using potassium as the activation medium gives 5.7 times the activation signal per unit velocity.

A number of competing reactions create potential interferences to detection of the ${ }^{38} \mathrm{~K} 2178 \mathrm{keV}$ gamma. The NIF target chamber is constructed of Al-5083, leaving potential interference from both $\mathrm{Al}$ and $\mathrm{Mg}$. The ${ }^{27} \mathrm{Al}(\mathrm{n}, \alpha)^{24} \mathrm{Na}$ reaction is known to be the primary contributor to the medium-term gamma dose and as such is the most likely to interfere with an activity measurement made in or around the target chamber. With a gamma ray energy of $2754 \mathrm{keV}$, the single-escape peak of ${ }^{24} \mathrm{Na}$ at $2243 \mathrm{keV}$ is likely to interfere with the ${ }^{38} \mathrm{~K}$ photopeak for a detector with limited energy resolution by making peak identification and fitting difficult to automate. As such, the system benefits significantly by using a lanthanum bromide scintillator over the less expensive sodium iodide, where the factor of two better resolution may improve peak discrimination while still allowing for use of a robust detector without the need for cryogenic cooling.

\section{ENGINEERING CONSIDERATIONS}

Radiation damage to electronics will be significant. Even beyond the target chamber wall, a detector system can expect a total neutron flow of greater than $10^{9}$ neutrons per shot, with an unknown energy spectrum highly-influenced by the unique mass configuration surrounding each detector. Hardware must withstand on order of 100 shots in order to be considered as economically viable. Chemical and physical properties of the activation medium become important to engineering design. This limits activation medium to a chemical form stable in air at $0-50$ ${ }^{\circ} \mathrm{C}$. The entire system must be practical to implement in the NIF environment. Detector electronics lifetimes are expected to limit overall hardware lifetime. Both $\mathrm{Zr}$ metal and potassium zirconate ceramic meet the materials properties requirements.

Detector and activation medium geometry are critical to the performance of the completed detector system. A well-type scintillator crystal surrounding the activation medium maximizes detection efficiency, but also maximizes background signal from the activated target chamber and other surrounding material. A cylindrical crystal completely surrounded by an activation medium shroud maximizes the relative signal from the activation medium to the near-environmental background, but gives a lower absolute count rate. A well detector filled with an activation medium and surrounded by a cylindrical shield jacket significantly lowers near-environmental background without sacrificing absolute detection efficiency, but the additional weight and complexity of the final assembly makes the detector more difficult to design, fabricate, and install on the NIF target chamber. Many different configurations would need detailed design analysis to determine the optimal design for any particular installation.

\section{CONCLUSION}

Installed detector systems would allow for measurement of short half-life activation reaction products and realize substantial improvement in capability within the NIF NAD system. The measurement of multiple reactions with different energy thresholds and cross section slopes near the neutron energy spectrum peak can discriminate core velocity and areal density effects. Both a potassium zirconate detector and a zirconium isomeric ratio detector may realize this potential improvement.

\section{REFERENCES}

${ }^{1}$ D.L. Bleuel et al., Rev. Sci. Instrum. these proceedings

${ }^{2}$ G.W. Cooper and C. L. Ruiz, Rev. Sci. Instrum. 72, 814 (2001).

${ }^{3}$ O. Landoas et al., Rev. Sci. Instrum. 82, 073501 (2011).

${ }^{4}$ B. M. Gatehouse and D. J. Lloyd, J. Solid State Chem. 2, 410 (1970).

${ }^{5}$ A. Abboud, P. Decowski, W. Grouchulski, A.

Marcinkowski, J. Piotrowski, K. Siwek, and Z. Wilhelmi, Nucl. Phys. A139, 42 (1969).

${ }^{6}$ Y. Kanda, Nucl. Phys. A185, 177 (1972).

${ }^{7}$ A. A. Filatenkov et al., Khlopin Radium Institute Report RI-252, St. Petersburg, 1999.

${ }^{8}$ A. Pavlik, G. Winkler, H. Vonach, A. Paulsen, and H.

Liskien, J. Phys. G: Nucl. Phys. 8, 1283 (1982). 

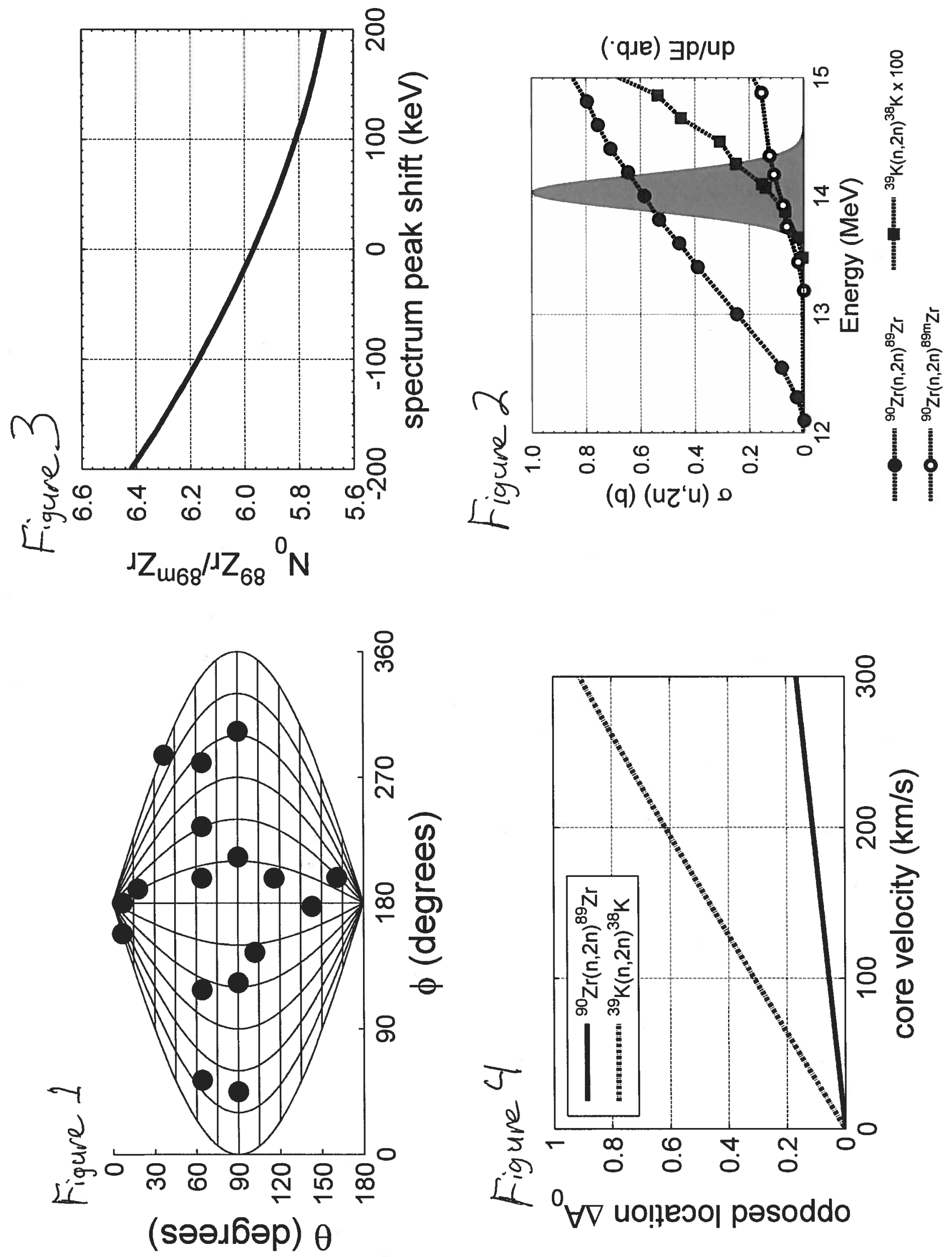